\title{
Erdős-Hajnal-type results on intersection patterns of geometric objects
}

\author{
Jacob Fox* János Pach ${ }^{\dagger}$
}

\begin{abstract}
In their seminal paper [21], Erdős and Hajnal raised the following question. Is it true that for any graph $G$ there exists a constant $c=$ $c(G)>0$ with the property that every graph of $n$ vertices that contains no induced subgraph isomorphic to $G$ has a complete or an empty induced subgraph of size $n^{c}$ ? We answer this question in the affirmative for some special classes of graphs defined by geometric methods.
\end{abstract}

\section{Introduction, definitions}

A classic result of Erdős and Szekeres [23] in Ramsey theory states that every graph on $n$ vertices contains a clique (that is, a complete subgraph) or an independent set of $\operatorname{size}^{1}$ at least $\frac{1}{2} \log n$. This bound, which has been slightly improved by Conlon [15], is tight up to a constant factor: Erdös [20] showed that there exists a graph on $n$ vertices, for every integer $n>1$, with no clique or independent of more than $2 \log n$ vertices.

Here we consider the same problem for intersection graphs of geometric objects. Given a system $\mathcal{S}$ of $n$ sets, their intersection graph is a graph $G_{\mathcal{S}}$ whose vertices are the elements of $\mathcal{S}$, two vertices $S, T \in \mathcal{S}$ being connected by an edge if and only if $S \cap T \neq \emptyset$. Applying the above theorem to $G_{\mathcal{S}}$, we obtain that $\mathcal{S}$ always contains at least $\frac{1}{2} \log n$ members that are either pairwise intersecting or pairwise disjoint. Can we say more than this, if we assume that the elements of $\mathcal{S}$ are "nice" geometric objects in some, say,

\footnotetext{
*Department of Mathematics, Princeton, Princeton, NJ. Email: jacobfox@math.princeton.edu. Supported by an NSF Graduate Research Fellowship and a Princeton Centennial Fellowship.

${ }^{\dagger}$ City College, CUNY and Courant Institute, NYU, New York, NY, USA. Email: pach@cims.nyu.edu. Supported by NSF Grant CCF-05-14079, and by grants from NSA, PSC-CUNY, the Hungarian Research Foundation OTKA, and BSF.

${ }^{1}$ All logarithms in this paper are of base two.
} 
Euclidean space? At first glance it seems that the answer is no. It was shown by Tietze [52] that every finite graph can be realized as the intersection graph of convex closed polytopes in $\mathbb{R}^{3}$, and we can even assume no two of these polytopes have an interior point in common. However, this statement is certainly not true in the plane. It is not hard to see that, for instance, the bipartite graph on 15 vertices formed by replacing each edge of the clique $K_{5}$ by a path of length 2 has no such realization [19]. (See Fig. 1.) This immediately implies that most graphs with $n$ vertices are not realizable in this way, as $n \rightarrow \infty$, since they almost surely contain a 15 -vertex induced subgraph isomorphic to the one depicted in Fig. 1.

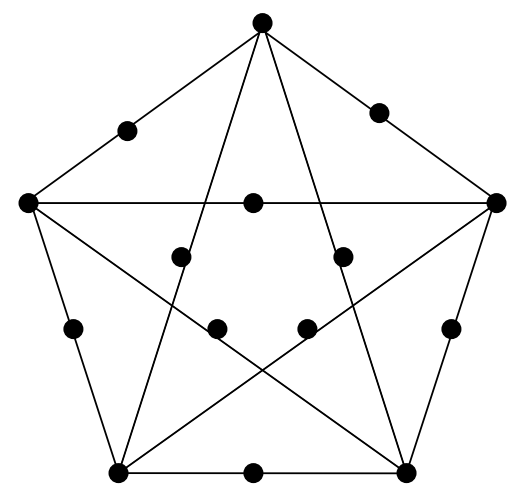

Figure 1: The fifteen vertex graph formed by replacing each edge of $K_{5}$ by a path of length two is not an intersection graph of connected sets in the plane.

In a seminal paper written in 1989, Erdős and Hajnal [21] showed that, given any graph $G$, the family $\mathcal{F}(G)$ of all graphs that do not contain $G$ as an induced subgraph have much stronger Ramsey-type properties than the family of all graphs. More precisely, they proved that there exists a constant $c=c(G)>0$, depending only on $G$, such that every graph of $n$ vertices that belongs to $\mathcal{F}(G)$ has a clique or an independent set of size at least $e^{c \sqrt{\log n}}$. They raised question whether one can always find a complete or empty induced subgraph of size $n^{c}$. This remains one of the most challenging open problems in Ramsey theory.

A complete bipartite graph is said to be balanced if its vertex classes differ in size by at most one. A balanced complete bipartite graph with $n$ vertices is called a bi-clique of size $n$.

Erdős, Hajnal, and Pach [22] proved a bipartite variant: There is a constant $c=c(G)>0$ such that every graph on $n$ vertices that belongs to 
$\mathcal{F}(G)$ or its complement contains a bi-clique of size $n^{c}$. Recently, Fox and Sudakov [30] strengthened this result: there is a constant $c=c(G)>0$ such that every graph on $n$ vertices that belongs to $\mathcal{F}(G)$ contains a bi-clique or an independent set of size $n^{c}$.

It is easy to see that the Erdös-Hajnal theorem generalizes to hereditary families of graphs, that is, to any family $\mathcal{F}$ (other than the family of all finite graphs) that is closed under taking induced subgraphs. The families of all graphs that can be realized as intersection graphs of connected sets, convex sets, disks, segments, etc. in the plane obviously belong to this category.

For convenience, we use the following terminology.

Definition: A family $\mathcal{F}$ of graphs has the

1. Erdös-Hajnal property if there is a constant $c(\mathcal{F})>0$ such that every graph in $\mathcal{F}$ on $n$ vertices contains a clique or an independent set of size $n^{c(\mathcal{F})}$;

2. strong Erdös-Hajnal property if there is a constant $b(\mathcal{F})>0$ such that for every graph $G$ in $\mathcal{F}$ on $n$ vertices, $G$ or its complement $\bar{G}$ contains a bi-clique of size $b(\mathcal{F}) n$.

The above terminology is justified by the following observation of Alon et $a l$. [7]: If a hereditary family of graphs has the strong Erdős-Hajnal property, then it also has the Erdős-Hajnal property.

To see this, we need the notion of cographs (or complement reducible graphs), also used by Erdős and Hajnal [16, 21]. The trivial graph with one vertex is a cograph, and so are the disjoint union and the join of two cographs. (The join can be obtained from the disjoint union by adding all edges between the two parts.)

Suppose now that $\mathcal{F}$ has the strong Erdös-Hajnal property with a constant $b>0$. That is, every $G \in \mathcal{F}$ with $n \geq 2$ vertices has two disjoint sets of vertices $V_{1}, V_{2}$, each of size at least $b n$, such that $G$ contains either all edges between $V_{1}$ and $V_{2}$ or no edges running between $V_{1}$ and $V_{2}$. Let $s(n)$ denote the largest number $s$ such that every $G \in \mathcal{F}$ with $n$ vertices contains a cograph with $s$ vertices. Applying the condition to the subgraphs of $G$ induced by $V_{1}$ and $V_{2}$, we obtain that $s(n) \geq 2 s(b n)$. Solving this recurrence, we conclude that $s(n) \geq n^{c}$, where $c=\frac{1}{\log 1 / b}$. It remains to notice that every cograph of $s$ vertices is a perfect graph, therefore it contains a clique or an independent set of size $\sqrt{s}$. Thus, $G$ or its complement has a clique of size at least $n^{c / 2}$, showing that $\mathcal{F}$ has the (weak) Erdős-Hajnal property.

It is certainly not true that all hereditary families of graphs have the strong Erdős-Hajnal property. For instance, the family of all triangle-free graphs does not have the strong Erdős-Hajnal property. 


\section{Convex sets and Dilworth's theorem}

It was shown by Larman et al. that the family of intersection graphs of plane convex sets has the Erdős-Hajnal property. In fact, a somewhat stronger statement is true. We call a connected set vertically convex if any vertical line intersects it in an interval.

Theorem 2.1 [41] Any family of $n$ vertically convex sets in the plane contains at least $n^{1 / 5}$ members that are either pairwise disjoint or pairwise intersecting.

For the proof of Theorem 2.1, we need Dilworth's theorem [17], according to which any partially ordered set of more than $p q$ elements contains a chain whose length is larger than $p$ or an antichain that has more than $q$ elements. Larman et al. [41] and Pach and Töröcsik [50] introduced four partial orders $<_{1},<_{2},<_{3},<_{4}$ on the family of all vertically convex sets in the plane such that any two disjoint sets are comparable with respect to at least one of these partial orders, but no two intersecting elements are. Applying Dilworth's theorem four times, we obtain that any family of $n$ plane convex sets has at least $n^{1 / 5}$ members that form a chain with respect to some $<_{i}$ or an antichain with respect to all $<_{i}(1 \leq i \leq 4)$. In the first case, these sets are pairwise disjoint, in the second case pairwise intersecting.

The best possible exponent in Theorem 2.1 is not known. Károlyi et al. [34] constructed a family of $n$ segments in the plane, which has no more than $n^{\log 4 / \log 27}$ members that are either pairwise disjoint or pairwise crossing. Recently, Jan Kynčl [40] has found a slightly better construction, for which the exponent is $\log 8 / \log 169$.

Dilworth's theorem implies that every partially ordered set of $n$ elements contains a chain or an antichain of size at least $\sqrt{n}$. If we have $r$ partial orders on the same $n$-element ground set, then, by repeated application of

Dilworth's theorem, there are at least $n^{\frac{1}{r+1}}$ elements such that no two are comparable by any of the $r$ orders, or we can choose one of the $r$ orders so that any two elements are comparable by it. Dumitrescu and G. Tóth [18] proved that for large values of $r$ this statement is not very far from being optimal.

Theorem 2.2 [18] For any $r \in \mathbb{N}$, there are $n$-element sets $P$ and $r$ partial orders defined on $P$ such that connecting two elements of $X$ if and only if they are comparable by at least one of these partial orders, the resulting graph contains neither a clique nor an independent set of size larger than $n^{\frac{1+\log r}{r+1}}$. 
The first named author established the existence of a much larger "homogeneous" bipartite pattern in partially ordered sets. For any partially ordered set $(P,>)$, we write $a \perp b$ if $a$ and $b$ are incomparable. For any pair of subsets $A$ and $B$ of $P$, we write $A>B$ if $a>b$ for all $a \in A$ and $b \in B$. Likewise, we write $A \perp B$ if $a \perp b$ for all $a \in A$ and $b \in B$.

Theorem 2.3 [24] Every n-element partially ordered set $(P,>)$ has two subsets $A, B \subset P$ with $|A|=|B| \geq \frac{n}{4 \log _{2} n}$ such that $A>B$ or $A \perp B$, provided that $n$ is sufficiently large. This result is tight up to a constant factor.

In another paper we generalized the last result to multiple partial orders.

Theorem 2.4 [25] Let $r$ be a fixed positive integer, and let $>_{1}, \ldots,>_{r}$ be partial orders on an $n$-element set $P$. Then there are two disjoint subsets

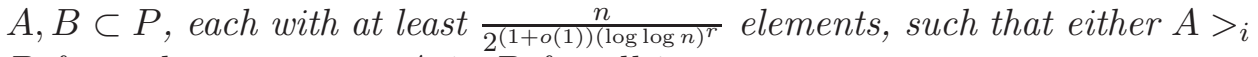
$B$ for at least one $i$, or $A \perp_{i} B$ for all $1 \leq i \leq r$.

Applying Theorem 2.4 to the $r=4$ partial orders defined on the family of vertically convex sets, which were mentioned above, we can conclude that any collection of $n$ vertically convex sets in the plane has two disjoint

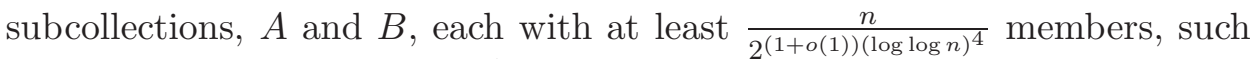
that either every member of $A$ intersects all members of $B$ or every member of $A$ is disjoint from all members of $B$. This is only slightly weaker than saying that the family of intersection graphs of vertically convex sets in the plane has the strong Erdős-Hajnal property. In fact, it was shown in [49] that this is not the case.

A continuous curve that intersects every vertical line in at most one point is called $x$-monotone. Obviously, every $x$-monotone curve is vertically convex. It is easy to see that the incomparability graph of every partially ordered set $(P,>)$, defined by connecting two elements of $P$ if and only if they are not comparable by $>$, can be realized as the intersection graph of a collection of $x$-monotone curves $[49,51]$. Therefore, it follows from the tightness of Theorem 2.3 that the family of intersection graphs of $x$ monotone curves does not have the strong Erdös-Hajnal property.

In [28], Theorem 2.3 was slightly strengthened as follows. There is a positive constant $c$ such that every $n$-element partially ordered set $(P,>)$ has two subsets $A, B \subset P$ such that either $|A|=|B| \geq \frac{c n}{\log n}$ and $A \perp B$, or $|A|=|B| \geq c n$ and $A>B$. For $x$-monotone curves, we have a similar result. 
Theorem 2.5 [28] There exists a constant $c>0$ with the property that the intersection graph $G$ of any collection of $n x$-monotone curves in the plane satisfies at least one of the following two conditions:

1. G contains a bi-clique of size $\frac{c n}{\log n}$; or

2. $\bar{G}$, the complement of $G$, contains a bi-clique of size cn.

For convex sets, we have a stronger result. In fact, it is as strong as it can get.

Theorem 2.6 [28] The family of intersection graphs of finite collections of convex sets in the plane has the strong Erdös-Hajnal property.

The proof relies on Turán-type results for incomparability graphs.

\section{Semialgebraic sets and Ramsey-graphs}

Pach and Solymosi [48] proved that the family of intersection graphs of line segments in the plane has the strong Erdős-Hajnal property. Later, Alon et al. [7] generalized this result to semialgebraic sets. To formulate this result more precisely, we need to agree about the definitions.

A semialgebraic set in $\mathbb{R}^{d}$ is the locus of all points that satisfy a given finite Boolean combination of polynomial equations and inequalities in the $d$ coordinates. We say that the description complexity of such a set $S$ is at most $\kappa$ if in some representation of $S$ the dimension $d$ is at most $\kappa$, the number of equations and inequalities is at most $\kappa$, and each of them has degree at most $\kappa$. (See [13].)

Every element $S$ of a family $\mathcal{F}$ of semialgebraic sets of constant description complexity $\kappa$ can be represented by a point $S^{*}$ of a $\kappa^{*}$-dimensional Euclidean space (in which the coordinates are, say, the coefficients of the monomials in the polynomials that define $S$ ). We say that a binary relation $R$ on $\mathcal{F} \times \mathcal{F}$ is semialgebraic, if the corresponding set

$$
\left\{\left(S^{*}, T^{*}\right) \in \mathbb{R}^{2 \kappa^{*}} \mid S, T \in \mathcal{F},(S, T) \in R\right\}
$$

is semialgebraic.

Theorem 3.1 [7] Let $\mathcal{F}$ be a family of semialgebraic sets of constant description complexity, and let $R \subseteq \mathcal{F} \times \mathcal{F}$ be a fixed semialgebraic relation on $\mathcal{F}$. Then there exists a constant $c>0$, which depends only on the maximum description complexity of the sets in $\mathcal{F}$ and of $R$, with the following 
property. Any collection of $n$ elements of $\mathcal{F}$ has two subcollections $\mathcal{F}_{1}$ and $\mathcal{F}_{2}$, each containing at least cn elements, such that either $\mathcal{F}_{1} \times \mathcal{F}_{2} \subseteq R$, or $\left(\mathcal{F}_{1} \times \mathcal{F}_{2}\right) \cap R=\emptyset$.

It is easy to verify that the relation that two sets $S, T \in \mathcal{F}$ has nonempty intersection is semialgebraic. Thus, Theorem 3.1 indeed implies that any family of intersection graphs of (real) semialgebraic sets of constant description complexity has the strong (and, therefore, the weak) Erdős-Hajnal property.

Corollary 3.2 For any family $\mathcal{F}$ of semialgebraic sets of constant description complexity, there is a constant $c=c(\mathcal{F})>0$ with the following property. Any collection of $n$ elements of $\mathcal{F}$ has two subcollections $\mathcal{F}_{1}$ and $\mathcal{F}_{2}$, each containing at least cn elements, such that either every element of $\mathcal{F}_{1}$ intersects all elements of $\mathcal{F}_{2}$, or no element of $\mathcal{F}_{1}$ intersects any element of $\mathcal{F}_{2}$.

Recently, Basu [12] has further extended this result for a broader class of algebraically defined sets.

Let us call an $n$-vertex graph $t$-Ramsey if it contains no clique and no independent set of size at least $t$. According to the results of Erdös [20] and Erdös and Szekeres [23] quoted in the introduction, there are $n$-vertex graphs that are $2 \log n$-Ramsey, but no $n$-vertex graph is $\frac{1}{2} \log n$-Ramsey. Erdős's construction is probabilistic, and it appears to be a formidable task to find comparably good efficient constructions. More precisely, there is no known polynomial time deterministic algorithm for the construction of $O(\log n)$-Ramsey graphs on $n$ vertices. Theorem 3.1 above shows that no such construction can be given by defining graphs using semialgebraic relations on a family of semialgebraic sets of constant description complexity. In fact, any $n$-vertex graph constructed in such a way will necessarily have a clique or an independent set of size at least $n^{c}$ for some $c>0$. This settles a conjecture of Babai [10], and improves a previous result [6] that showed that such graphs cannot be $t$-Ramsey for $t=e^{o(\sqrt{\log n})}$. The best known polynomial time construction is due to Barak et al. [11] and it produces $2^{(\log n)^{o(1)}}$-Ramsey graphs. The previous record was held by Frankl and Wilson [31].

As we remarked in the Introduction, in three and higher dimensions the family of intersection graphs of convex sets does not have the Erdös-Hajnal property. Somewhat surprisingly, Corollary 3.2 holds in any fixed dimension. There is another special class of intersection graphs of higher dimensional 
convex bodies that has the Erdős-Hajnal property. A set $S \subset \mathbb{R}^{d}$ is called $K$-fat if there are two $d$-dimensional balls $B_{1}$ and $B_{2}$ such that $B_{1} \subseteq S \subseteq B_{2}$ and the ratio of the radius of $B_{2}$ to the radius of $B_{1}$ is at most $K$.

Theorem 3.3 For any constant $K \geq 1$ and for any positive integer $d$, the family of intersection graphs of $K$-fat convex bodies in $\mathbb{R}^{d}$ has the strong Erdös-Hajnal property.

Theorem 3.3 can be strengthened to say that there is a constant $c=$ $c(K, d)>0$ such that every intersection graph of $K$-fat convex bodies in $\mathbb{R}^{d}$ contains a clique or its complement contains a bi-clique of size $\mathrm{cn}$. One proof of this result uses the separator theorem of Miller, Teng, Thurston, and Vavasis [45] discussed in the next section. Theorem 3.3 does not remain true for arbitrary (not necessarily convex) $K$-fat bodies even in the case $d=2$. Theorem 3.1 can be proved by a standard linearization process (see e.g. [3]) to transform the elements of $\mathcal{F}$ into vectors in a higher dimensional space, and the relation $R$ to the set of all pairs of vectors $(u, v)$ with a nonnegative scalar product $\langle u, v\rangle$. Thus, Theorem 3.1 can be reduced to the following

Lemma 3.4 [7] Let $U$ and $V$ be finite multisets of vectors in $\mathbb{R}^{d}$. Then there are subsets $U^{\prime} \subset U$ and $V^{\prime} \subset V$ such that $\left|U^{\prime}\right| \geq \frac{1}{2^{d+1}}|U|,\left|V^{\prime}\right| \geq \frac{1}{2^{d+1}}|V|$, and either $\langle u, v\rangle \geq 0$ for all $u \in U^{\prime}, v \in V^{\prime}$, or $\langle u, v\rangle<0$ for all $u \in U^{\prime}$, $v \in V^{\prime}$.

For $d>2$, in spite of the apparent simplicity of Lemma 3.4, we do not have any "elementary" proof that would avoid using the probabilistic method or some form of the Borsuk-Ulam theorem (specifically, a partition theorem of Yao and Yao [53]). We challenge the reader to come up with such a proof.

\section{String graphs and separator theorems}

A graph that can be realized as the intersection graph of finitely many continuous curves (strings) in the plane is called a string graph. As it was pointed out toward the end of Section 2, the family of intersection graphs of $x$-monotone curves, and thus the family of string graphs, do not have the strong Erdős-Hajnal property. However, it was conjectured in [49], that the situation is different if we restrict our attention to collections of curves with a bounded number of intersections per pair.

A collection of curves in the plane is called t-intersecting if any two of them intersect in at most $t$ points. The elements of a 1 -intersecting 
collection of curves are called pseudo-segments. A collection of portions of algebraic curves of maximum degree $d$ in general position is $d^{2}$-intersecting. Clearly, the intersection graphs of $t$-intersecting collections of curves form a hereditary family.

Theorem 4.1 [29] For every $t \in \mathbb{N}$, the family of intersection graphs of t-intersecting collections of curves in the plane has the strong Erdös-Hajnal property. That is, for every $t \in \mathbb{N}$, there is a constant $c_{t}>0$ such that the intersection graph $G$ of every t-intersecting collection of $n$ curves in the plane contains a bi-clique of size $c_{t} n$ or its complement $\bar{G}$ contains a bi-clique of size $c_{t} n$.

For the proof, we need to extend the Lipton-Tarjan separator theorem [42].

A separator for a graph $G=(V, E)$ is a subset $V_{0} \subset V$ such that there is a partition $V=V_{0} \cup V_{1} \cup V_{2}$ with $\left|V_{1}\right|,\left|V_{2}\right| \leq \frac{2}{3}|V|$ and no vertex in $V_{1}$ is adjacent to any vertex in $V_{2}$. The Lipton-Tarjan separator theorem states that every planar graph with $n$ vertices has a separator of size $O(\sqrt{n})$. By an important theorem of Koebe [37], every planar graph can be represented as the intersection (incidence) graph of nonoverlapping closed disks in the plane. Miller, Teng, Thurston, and Vavasis [45] proved that for every $d \geq 2$, the intersection graph of any collection of $n$ balls in $\mathbb{R}^{d}$ such that no point belongs to more than $k$ of them has a separator of size $O\left(d k^{1 / d} n^{1-1 / d}\right)$.

A Jordan region is a subset of the plane that is homeomorphic to a closed disk. We say that a Jordan region $R$ contains another Jordan region $S$ if $S$ lies in the interior of $R$. A crossing between $R$ and $S$ is either a crossing between their boundaries or a containment between them. The following result is a generalization of the separator theorems of Lipton and Tarjan and of Miller, Teng, Thurston, and Vavasis [45] in two dimensions.

Theorem 4.2 [26] If $C$ is a finite collection of Jordan regions with a total of $m$ crossings, then the intersection graph of $C$ has a separator of size $O(\sqrt{m})$.

By slightly fattening curves in the plane, Theorem 4.2 implies that it is also true for curves in the plane instead of Jordan regions. The proof of Theorem 4.1 has another interesting feature. The statement guarantees the existence of a large bi-clique in the graph $G$ or in its complement $\bar{G}$. As it turns out, in many cases it can be proved that both $G$ and $\bar{G}$ contain large bi-cliques. If $G$ is a "dense" graph, then it must contain a bi-clique. Otherwise, $\bar{G}$ contains a bi-clique. 
Theorem 4.3 [29] Let $C$ be a t-intersecting collection of $n$ curves in the plane such that at least $\varepsilon n^{2}$ pairs of them intersect. Then the intersection graph of $C$ contains a bi-clique of size at least $c_{t} \varepsilon^{64} n$, where $c_{t}>0$ depends only on $t$.

Fox et al. [29] have also generalized Theorem 4.1 in another direction. We first need a few definitions. Define an $r$-region to be a subset of the plane that is union of at most $r$ Jordan regions. Call these (at most $r$ ) Jordan regions of an $r$-region the components of the $r$-region. A family of Jordan regions is t-intersecting if the boundaries of any two of them intersect in at most $t$ points. A collection of $r$-regions is $t$-intersecting if the collection of all of its components is $t$-intersecting. They showed that for all $r, t \in \mathbb{N}$, the family of intersection graphs of finite collections of $t$-intersecting families of $r$-regions has the strong Erdős-Hajnal property.

Note that the last result can also be regarded as a generalization of Corollary 3.2 in the planar case, which states that the family of intersection graphs of collections of semialgebraic sets of constant description complexity has the strong Erdös-Hajnal property. Indeed, the boundary of a semialgebraic set of bounded description complexity in the plane is the union of a bounded number of algebraic curves of bounded degree, any two of which either intersect in a bounded number of points or overlap. By slightly perturbing semialgebraic sets, while maintaining their intersection pattern and their description complexity, we can assume that the boundaries of no two semialgebraic sets overlap. We can further assume, by slightly fattening the

sets, if necessary, that each of them is the union of a constant number of Jordan regions, so that the above result applies.

In Table 1, we summarize the discussed results concerning Erdős-Hajnal properties for various families of intersection graphs.

\section{$5 \quad$ Asymmetric Ramsey-type questions}

So far we discussed a variety of results that guarantee the existence of unexpectedly large homogeneous (sometimes bipartite) subgraphs in intersection graphs of various geometric objects. These results were symmetric, in the sense that in most of them empty and complete subgraphs played symmetric roles. In the spirit of so-called "off-diagonal" Ramsey theory, we can consider asymmetric variants of these questions.

A classical asymmetric result is the following theorem of Ajtai, Komlós, and Szemerédi [4]: Every triangle-free graph on $n$ vertices contains an independent set of size $\Omega(\sqrt{n \log n})$. Kim [35] proved that this bound is tight up 
Table 1: Erdős-Hajnal properties

\begin{tabular}{crr}
\hline \hline & & \\
family of intersection graphs of & $\begin{array}{r}\text { Erdős-Hajnal } \\
\text { Property }\end{array}$ & $\begin{array}{r}\text { Erdös-Hajnal } \\
\text { Property }\end{array}$ \\
\hline \hline convex sets in $\mathbb{R}^{3}$ & no & no \\
\hline fat convex sets in $\mathbb{R}^{d}$ & yes & yes \\
\hline convex sets in $\mathbb{R}^{2}$ & yes & yes \\
\hline$x$-monotone curves in $\mathbb{R}^{2}$ & yes & no \\
\hline curves in $\mathbb{R}^{2}$ & $?$ & no \\
\hline$t$-intersecting collections of \\
curves in $\mathbb{R}^{2}$ & yes & yes \\
\hline$t$-intersecting collections of & & yes \\
$r$-regions in $\mathbb{R}^{2}$ & yes & yes \\
\hline semialgebraic sets of & & nes \\
constant description complexity & & ? \\
\hline fat connected sets in $\mathbb{R}^{2}$ & &
\end{tabular}

to a constant factor. If we restrict our attention to certain types of planar intersection graphs, this bound can be substantially improved.

Theorem 5.1 [27] If $G$ is a $K_{k}$-free intersection graph of a t-intersecting family of $n \geq k$ curves in the plane, then $G$ contains an independent set of size at least $n\left(c_{t} \frac{\log k}{\log n}\right)^{c \log k}$, where $c$ is an absolute constant and $c_{t}>0$ only depends on $t$.

Taking $\delta$ such that $\epsilon=c \delta \log \frac{1}{c_{t} \delta}$, we have the following corollary.

Corollary 5.2 [27] For each $\epsilon>0$ and positive integer $t$, there is $\delta=$ $\delta(\epsilon, t)>0$ such that if $G$ is an intersection graph of a $t$-intersecting family of $n$ curves in the plane, then $G$ has a clique of size at least $n^{\delta}$ or an independent set of size at least $n^{1-\epsilon}$.

Note that Corollary 5.2 is stronger than saying that the family of intersection graphs of $t$-intersecting families of curves in the plane has the Erdős-Hajnal property. 
By slightly fattening curves in the plane, it is easy to see that if $G$ is an intersection graph of a $t$-intersecting collection of curves, then $G$ is also an intersection graph of a $4 t$-intersecting collection of Jordan regions.

As usual, let $\chi(G)$ and $\alpha(G)$ denote the chromatic number and the size of the largest independent set of a graph $G$. Clearly, we have $\alpha(G) \geq \frac{n}{\chi(G)}$. It is not hard to generalize Theorem 5.1, as follows.

Theorem 5.3 [27] If $G$ is a $K_{k}$-free intersection graph of a t-intersecting family of $n$ r-regions, then

$$
\chi(G) \leq\left(c_{t, r} \frac{\log n}{\log k}\right)^{c r \log k},
$$

where $c_{t, r}$ only depends on $t$ and $r$ and $c$ is an absolute constant.

In the plane, every semialgebraic set of constant description complexity is the intersection graph of a $t$-intersecting collection of $r$-regions, where $r$ and $t$ depend only on the description complexity. Therefore, we have the following corollary of Theorem 5.3.

Corollary 5.4 [27] If $G$ is a $K_{k}$-free intersection graph of a collection of semialgebraic sets in the plane of description complexity $d$, then

$$
\chi(G) \leq\left(c_{d} \frac{\log n}{\log k}\right)^{c_{d} \log k},
$$

where $c_{d}$ only depends on $d$.

A pair of convex sets or a pair of $x$-monotone curves can have arbitrarily many intersection points between their boundaries. Thus, Theorem 5.3 is not directly applicable to their intersection graphs. Nevertheless, we can show the following result.

Theorem 5.5 [27] If $G$ is a $K_{k}$-free intersection graph of $n$ convex sets in the plane, then

$$
\chi(G) \leq\left(c \frac{\log n}{\log k}\right)^{13 \log k},
$$

where $c$ is an absolute constant.

Taking $\delta$ such that $\epsilon=13 \delta \log \frac{c}{\delta}$, and noting that $\alpha(G) \geq \frac{n}{\chi(G)}$, for every graph $G$ with $n$ vertices, we obtain the following corollary of Theorem 5.5. 
Corollary 5.6 [27] For each $\epsilon>0$ there is $\delta=\delta(\epsilon)>0$ such that every intersection graph of $n$ convex sets in the plane has a clique of size at least $n^{\delta}$ or an independent set of size at least $n^{1-\epsilon}$.

A more general form of Theorem 2.1 states that, for every positive integer $k$, every family of $n$ convex sets in the plane has an independent set of size $k$ or a clique of size at least $n / k^{4}[41]$. Notice that Corollary 5.6 only applies in the case that the clique number is not too large while the result of Larman et al. [41] only applies when the independence number is not too large.

We can also prove the following theorem.

Theorem 5.7 [27] If $G$ is a $K_{k}$-free intersection graph of $n x$-monotone curves in the plane, then

$$
\chi(G) \leq(c \log n)^{15 \log k},
$$

where $c$ is an absolute constant.

A collection $C$ of curves in the plane is grounded if there is a closed (Jordan) curve $\gamma$ such that every curve in $C$ has one endpoint on $\gamma$ and the rest of the curve lies in the exterior of $\gamma$. The intersection graph of a collection of grounded curves is called an outerstring graph.

McGuinness [43] proved that there is a constant $C$ such that if $G$ is a triangle-free intersection graph of a grounded 1-intersecting collection of curves, then $G$ has chromatic number at most $C$. In Section 7 , we prove an upper bound for the chromatic number of $K_{k}$-free outerstring graphs.

A survey by Kostochka [38] discusses results on coloring intersection graphs of certain geometric figures. Some of the known bounds are summarized in Table 5.

In the next two sections, we illustrate some of the ideas used for establishing the above results from [27] by giving simple proofs of some weaker versions of Theorems 5.1 and 5.7.

\section{Independent sets in string graphs}

Let $I_{t}(n, k)$ denote the maximum $I$ such that every $K_{k}$-free intersection graph of $n$ curves in the plane with no pair of curves intersecting in more than $t$ points has an independent set of size $I$. The aim of this section is to establish some weaker versions of Theorem 5.1.

We first prove a very simple lower bound for $I_{t}(n, k)$, and then we show how to improve it with a little extra care. 
Table 2: Chromatic numbers of $K_{k}$-free intersection graphs

\begin{tabular}{crr}
\hline \hline $\begin{array}{c}K_{k} \text {-free } \\
\text { intersection graphs of }\end{array}$ & $\begin{array}{r}\text { upper bound on } \\
\text { chromatic number }\end{array}$ & reference \\
\hline \hline intervals in $\mathbb{R}$ & $k-1$ & Gallai, Hajós \\
\hline arcs along a circle & $\left\lfloor\frac{3(k-1)}{2}\right\rfloor$ & Karapetian [32] \\
\hline segments in $\mathbb{R}^{2}$ & $?$ & Erdös \\
\hline half-lines in $\mathbb{R}^{2}, k=3$ & $<\infty$ & Karapetian [33] \\
\hline chords of a circle, $k=3$ & $50 \cdot 2^{k}$ & Kostochka-Kratochvíl [39] \\
\hline chords of a circle & $3 k^{2}-8 k+4$ & Asplund, Grünbaum [9] \\
\hline $\begin{array}{c}\text { axis-parallel } \\
\text { rectangles in } \mathbb{R}^{2}\end{array}$ & & and C. Hendler \\
\hline unit squares in $\mathbb{R}^{2}, k=3$ & $3 \mathrm{k}-6$ & Akiyama et al. [5] \\
\hline translates of convex & & Kim et al. [36] \\
body in $\mathbb{R}^{2}$ & $(O(d))^{d} k$ & Pach [46] and \\
\hline $\begin{array}{c}\text { homothetic copies of } \\
\text { convex body in } \mathbb{R}^{d}\end{array}$ & & Kostochka [38] \\
\hline $\begin{array}{c}\text { axis-parallel } \\
\text { boxes in } \mathbb{R}^{d}, d \geq 3\end{array}$ & $\infty$ & Burling [14] \\
\hline
\end{tabular}

Proposition 6.1 There is an absolute constant $c$ such that for all positive integers $n, k, t$ with $n, k \geq 2$, we have $I_{t}(n, k) \geq \frac{n}{\left(c t^{1 / 2} \log n\right)^{2(k-2)}}$. That is, every $K_{k}$-free intersection graph of $n$ curves in the plane with no pair intersecting in more than $t$ points has an independent set of size at least $\frac{n}{\left(c t^{1 / 2} \log n\right)^{2(k-2)}}$.

Proof: The proof is by induction on $n$ and $k$. The base cases $n=2$ and $k=2$ are trivial. Let $G$ be a $K_{k}$-free intersection graph of a $t$ intersecting collection $C$ of $n$ curves in the plane such that the largest independent set in $G$ has size $I_{t}(n, k)$. If there is a vertex $v$ adjacent to at least $n\left(c t^{1 / 2} \log n\right)^{-2}$ other vertices, then the intersection graph of the neighborhood of $v$ has no clique of size $k-1$, and by induction, we are done in this case. So we may assume that the maximum degree of $G$ is at most $n\left(c t^{1 / 2} \log n\right)^{-2}$. The number of crossings between elements of $C$ is at most $\frac{1}{2} t n^{2}\left(c t^{1 / 2} \log n\right)^{-2}<n^{2}(c \log n)^{-2}$. Applying the separator theorem for curves, which is a corollary of Theorem 4.2, there is a partition $C=C_{0} \cup C_{1} \cup C_{2}$, with $\left|C_{0}\right|<c^{\prime} n / \log n,\left|C_{1}\right|,\left|C_{2}\right| \leq 2 n / 3$, and 
no edges between $C_{1}$ and $C_{2}$ in $G$, where $c^{\prime}$ is $\frac{1}{c}$ times the implied constant in the separator theorem. Letting $a_{1}=\left|C_{1}\right|$ and $a_{2}=\left|C_{2}\right|$, we have $a_{1}+a_{2} \geq n-c^{\prime} n / \log n, a_{1}, a_{2} \leq 2 n / 3$, and

$$
I_{t}(n, k) \geq I_{t}\left(a_{1}, k\right)+I_{t}\left(a_{2}, k\right) .
$$

Using the induction hypothesis, we have

$$
I_{t}\left(a_{i}, k\right) \geq a_{i}^{2}\left(c t^{1 / 2} \log a_{i}\right)^{-2(k-2)}
$$

for $i=1,2$. It is straightforward to check that we can pick $c$ large enough so that $c^{\prime}$ is small enough so that and combining (1) and (2) gives the desired lower bound on $I_{t}(n, k)$.

By also keeping track of the number of edges of the intersection graph $G$, we can improve the exponent of the $\log n$ factor in the lower bound in Proposition 6.1 from $2(k-2)$ to $k-2$.

Proposition 6.2 There is an absolute constant $c$ such that for all positive integers $n, k, t$ with $n, k \geq 2$, we have $I_{t}(n, k) \geq \frac{n}{(\operatorname{ct} \log n)^{(k-2)}}$.

Proposition 6.2 follows from the next statement, by induction on $k$; the base case $k=2$ is trivial.

Proposition 6.3 There is a constant $c$ such that if $G$ is a nonempty intersection graph of a t-intersecting collection of $n \geq 2$ curves in the plane, then $G$ contains an induced subgraph with at least $\frac{c n}{t \log n}$ vertices whose clique number is strictly less than the clique number of $G$.

Proof: Let $D_{t}(m, n)$ denote the maximum $D$ such that every graph $G$ with $n$ vertices and $m \geq 1$ edges, which is an intersection graph of a $t$-intersecting collection of curves in the plane, has an induced subgraph with $D$ vertices such that its clique number is strictly smaller than the clique number of $G$.

It is sufficient to show that there is a constant $c$ such that

$$
D_{t}(m, n) \geq \frac{c n}{t \log n}+\frac{m}{n},
$$

for all $m$ and $n$ with $n \geq 2$.

The proof is by induction on $n$, noting that $D_{t}(0,1)=1$. Let $G$ be an intersection graph of a $t$-intersecting collection $C$ of curves in the plane with $n$ vertices, $m$ edges, and every induced subgraph of $G$ of size larger than $D_{t}(m, n)$ has the same clique number as $G$. Let $\Delta$ be the maximum 
degree of $G$. Notice that $\Delta \leq D_{t}(m, n)$ since the induced subgraph by the neighborhood of a vertex of maximum degree has clique number less than the clique number of $G$. Also $\Delta \geq 2 m / n$ since $2 m / n$ is the average degree of $G$. Hence, if $\Delta \geq 2 \frac{c n}{t \log n}$, then the desired inequality holds. Therefore, we may assume $\Delta<2 \frac{c n}{t \log n}$.

By Theorem 4.2, the separator theorem, there is a partition $C=C_{0} \cup$ $C_{1} \cup C_{2}$ with $\left|C_{0}\right|<c^{\prime} t \sqrt{m},\left|C_{1}\right|,\left|C_{2}\right| \leq 2 n / 3$ and no curve in $C_{1}$ intersects a curve in $C_{2}$, where $c^{\prime}$ is the implied constant for the separator theorem. For $i \in\{1,2\}$, let $n_{i}$ and $m_{i}$ denote the number of vertices and edges, respectively, of the subgraph of $G$ induced by $C_{i}$. So

$$
D_{t}(m, n) \geq D_{t}\left(m_{1}, n_{1}\right)+D_{t}\left(m_{2}, n_{2}\right),
$$

with

$$
\begin{gathered}
n_{1}, n_{2} \leq 2 n / 3, \\
n_{1}+n_{2} \geq n-\left|C_{0}\right| \geq n-c^{\prime} t \sqrt{m},
\end{gathered}
$$

and

$$
m_{1}+m_{2} \geq m-\Delta\left|C_{0}\right| \geq m-2 \frac{c n}{t \log n} c^{\prime} t \sqrt{m}=m-2 c c^{\prime} \frac{n}{\log n} \sqrt{m} .
$$

Notice that, by the induction hypothesis,

$$
\begin{gathered}
D_{t}(m, n) \geq D_{t}\left(m_{1}, n_{1}\right)+D_{t}\left(m_{2}, n_{2}\right) \geq \frac{c n_{1}}{t \log n_{1}}+\frac{m_{1}}{n_{1}}+\frac{c n_{2}}{t \log n_{2}}+\frac{m_{2}}{n_{2}} \\
\geq \frac{c\left(n_{1}+n_{2}\right)}{t \log (2 n / 3)}+\frac{m_{1}+m_{2}}{2 n / 3} \geq \frac{c\left(n_{1}+n_{2}\right)}{t(-1 / 2+\log n)}+\frac{m_{1}+m_{2}}{2 n / 3} .
\end{gathered}
$$

Case 1: $m \geq\left(\frac{12 c c^{\prime}}{t} \frac{n}{\log n}\right)^{2}$. In this case,

$$
m_{1}+m_{2} \geq m-2 c c^{\prime} \frac{n}{\log n} \sqrt{m} \geq 5 m / 6 .
$$

Also, using (3),

$$
\begin{gathered}
D_{t}(m, n) \geq \frac{c\left(n_{1}+n_{2}\right)}{t \log n}+\frac{5}{4} m / n=\frac{c n}{t \log n}+\frac{m}{n}+\frac{m}{4 n}-\frac{c\left(n-\left(n_{1}+n_{2}\right)\right)}{t \log n} \\
\geq \frac{c n}{t \log n}+\frac{m}{n}+\frac{m}{4 n}-\frac{c c^{\prime} \sqrt{m}}{t \log n} \geq \frac{c n}{t \log n}+\frac{m}{n},
\end{gathered}
$$

completing the analysis in this case. 
Case 2: $m<\left(\frac{12 c c^{\prime}}{t} \frac{n}{\log n}\right)^{2}$. Using (3), we have

$$
\begin{gathered}
D_{t}(m, n) \geq \frac{c\left(n_{1}+n_{2}\right)}{t(-1 / 2+\log n)} \geq \frac{c}{t}\left(n-c^{\prime} t \sqrt{m}\right)\left(\frac{1}{\log n}+\frac{1}{2 \log ^{2} n}\right) \\
\geq \frac{c}{t}\left(\frac{n}{\log n}+\frac{n}{2 \log ^{2} n}-\frac{2 c^{\prime} t \sqrt{m}}{\log n}\right) \geq \frac{c n}{t \log n}+\frac{m}{n} .
\end{gathered}
$$

as long as $c$ is chosen originally to be at most $\frac{1}{576 c^{\prime 2}}$. This completes the proof.

\section{$7 \quad$ Independent sets of $x$-monotone curves}

In this section, we prove a weaker version of Theorem 5.7.

If a graph has small chromatic number, then it has a large independent set. The following result is an analogue of Proposition 6.1 for intersection graphs of $x$-monotone curves.

Proposition 7.1 There is a constant c such that every intersection graph of $n x$-monotone curves with no $k>2$ pairwise crossing has chromatic number at most $(c \log n)^{2 k-3}$.

Let $X(n, k)$ denote the maximum chromatic number over all $K_{k}$-free intersection graphs of $n x$-monotone curves. Let $V(n, k)$ denote the maximum chromatic number over all $K_{k}$-free intersection graphs of $n x$-monotone curves that each intersect a fixed vertical line $L$. We start the proof of Proposition 7.1 with the following lemma relating $V(n, k)$ and $X(n, k)$.

Lemma 7.2 For all positive integers $n$ and $k$, we have

$$
X(n, k) \leq X\left(\left\lfloor\frac{n}{2}\right\rfloor, k\right)+V(n, k) .
$$

Proof: Let $C$ be a family of $n x$-monotone curves, and let $x_{1} \leq \ldots \leq x_{n}$ be the $x$-coordinates of the left endpoints of the $n x$-monotone curves. Let $L$ be the vertical line $x=x_{\left\lceil\frac{n}{2}\right\rceil}$. Notice that every $x$-monotone curve whose right endpoint has $x$-coordinate less than $x_{\left\lceil\frac{n}{2}\right\rceil}$ is disjoint from every $x$ monotone curve whose left endpoint has $x$-coordinate more than $x_{\left\lceil\frac{n}{2}\right\rceil}$. There are at most $\left\lfloor\frac{n}{2}\right\rfloor$ curves whose right endpoint has $x$-coordinate less than $x_{\left\lceil\frac{n}{2}\right\rceil}$ and at most $\left\lfloor\frac{n}{2}\right\rfloor$ curves whose left endpoint has $x$-coordinate greater than $x_{\left\lceil\frac{n}{2}\right\rceil}$. Hence, we can properly color the $x$-monotone curves in $C$ that 
do not intersect $L$ with $X\left(\left\lfloor\frac{n}{2}\right\rfloor, k\right)$ colors. We can color the remaining $x$ monotone curves in $C$, which all intersect $L$, with $V(n, k)$ colors. Hence, $X(n, k) \leq X\left(\left\lfloor\frac{n}{2}\right\rfloor, k\right)+V(n, k)$.

By iterating Lemma 7.2, we obtain

$$
X(n, k) \leq \sum_{i=0}^{\lfloor\log n\rfloor} V\left(\left\lfloor\frac{n}{2^{i}}\right\rfloor, k\right) \leq\left(1+\log _{2} n\right) V(n, k) .
$$

Recall that a collection $C$ of curves in the plane is grounded if there is a closed (Jordan) curve $\gamma$ such that every curve in $C$ has one endpoint on $\gamma$ and the rest of the curve lies in the exterior of $\gamma$. Also recall that the intersection graph of a collection of grounded curves is called an outerstring graph. Let $G(n, k)$ denote the maximum chromatic number over all $K_{k}$-free outerstring graphs with $n$ vertices. The following lemma relates $G(n, k)$ and $V(n, k)$.

Lemma 7.3 For all positive integers $n$ and $k$, we have

$$
V(n, k) \leq G(n, k)^{2} .
$$

Proof: Let $C=\left\{C_{1}, \ldots, C_{n}\right\}$ be a family of $n x$-monotone curves that intersect a vertical line $L: x=x_{0}$. Let $L_{i}$ denote the intersection of $C_{i}$ with the left half-plane $\left\{(x, y): x \leq x_{0}\right\}$. Let $R_{i}$ denote the intersection of $C_{i}$ with the right half-plane $\left\{(x, y): x \geq x_{0}\right\}$. Let $\mathcal{L}=\left\{L_{1}, \ldots, L_{n}\right\}$ and $\mathcal{R}=\left\{R_{1}, \ldots, R_{n}\right\}$. Notice that the intersection graph of $\mathcal{L}$ can be properly colored with $G(n, k)$ colors, and the intersection graph of $\mathcal{R}$ can be properly colored with $G(n, k)$ colors. Consider proper colorings $c_{1}$ : $\mathcal{L} \rightarrow\{1, \ldots, G(n, k)\}$ and $c_{2}: \mathcal{R} \rightarrow\{1, \ldots, G(n, k)\}$ of the intersection graphs of $\mathcal{L}$ and $\mathcal{R}$, respectively. Assign to each $x$-monotone curve $C_{i}$ the color $\left(c_{1}\left(L_{i}\right), c_{2}\left(R_{i}\right)\right)$. The family $\mathcal{C}$ is properly colored with $G(n, k)^{2}$ colors. Hence, $V(n, k) \leq G(n, k)^{2}$.

A family $C$ of $n$ grounded curves naturally comes with a cyclic labeling by their endpoints along the ground. Start by assigning any grounded curve the label 0 and then proceed to label the grounded curves clockwise, breaking ties arbitrarily, so the $(i+1)^{t h}$ grounded curve has label $i$. The labels are elements of $\mathbb{Z}_{n}$. Define the distance between a pair of grounded curves in $C$ as the cyclic distance between their labels, that is, the distance $d(i, j)$ between the arc with label $i$ and the arc with label $j$ is $\min (|i-j|, n-|i-j|)$. We let $[i, j]$ denote the cyclic interval of elements $\{i, i+1, \ldots, j\}$.

The following is the main lemma of this section. 
Lemma 7.4 For all integers $n \geq 2$ and $k \geq 3$, we have $G(n, k) \leq G\left(\left\lfloor\frac{2 n}{3}\right\rfloor, k\right)+$ $4 G(n, k-1)$.

Proof: Let $C=\left\{C_{1}, \ldots, C_{n}\right\}$ be a family of $n \geq 2$ grounded curves with curve $C_{i}$ having cyclic label $i$. If no pair of $\operatorname{arcs}$ in $C$ intersect, then the chromatic number of the intersection graph of $C$ is 1 . Therefore, we may suppose that there are a pair of curves in $C$ that intersect.

Let $\left(C_{a}, C_{b}\right)$ be a pair of arcs that intersect such that the distance $d(a, b)$ is the maximum distance over all pairs of curves in $C$ that intersect.
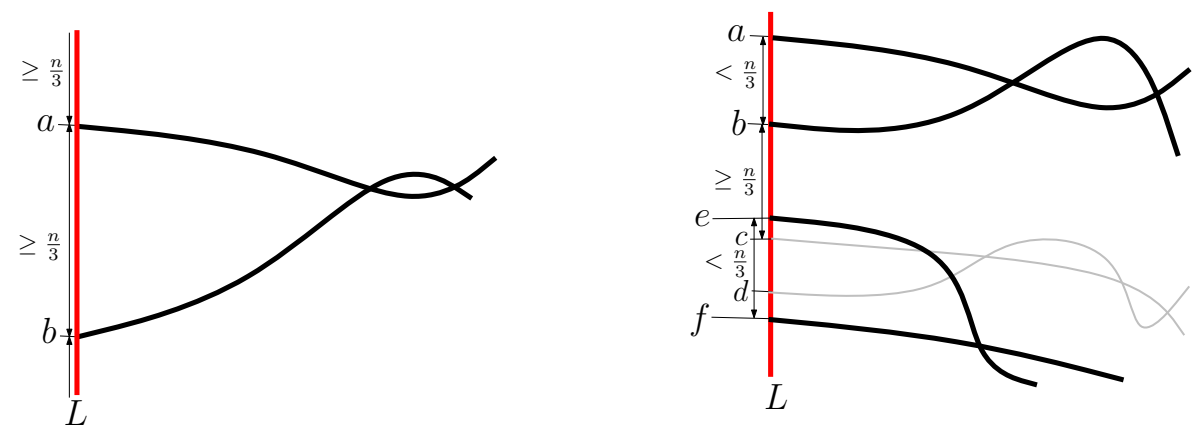

Figure 2: On the left: there are two curves, $C_{a}$ and $C_{b}$, that intersect and whose cyclic distance along $L$ is at least $n / 3$. On the right: the maximum distance between any two curves that intersect is less than $n / 3$.

Case 1: $d(a, b) \geq \frac{n}{3}$ (which is depicted in the left-hand side of Figure 2. If $C_{i}$ with $i$ in the cyclic interval $[a+1, b-1]$ is disjoint from $C_{a}$ and $C_{b}$, and $C_{j}$ with $j$ in the cyclic interval $[b+1, a-1]$ is disjoint from $C_{a}$ and $C_{b}$, then $C_{i}$ and $C_{j}$ are disjoint. Hence, the curves that are disjoint from $C_{a}$ and $C_{b}$ can be properly colored with $G(n-d(a, b)-1, k) \leq G\left(\left\lfloor\frac{2 n}{3}\right\rfloor-1, k\right)$ colors. We can properly color the curves in $C$ that intersect $C_{a}$ with $G(n, k-1)$ colors, and properly color the curves in $C$ that intersect $C_{b}$ with $G(n, k-1)$ colors. Therefore, $C$ can be properly colored with $G\left(\left\lfloor\frac{2 n}{3}\right\rfloor-1, k\right)+2 G(n, k-1)$ colors.

Case 2: $d(a, b)<\frac{n}{3}$ (which is depicted in the right-hand side of Figure 2. Let $c \in \mathbb{Z}_{n}$ be given by $c \equiv b+\left\lceil\frac{n}{3}\right\rceil(\bmod n)$. If the curve $C_{c}$ is disjoint from the other arcs in $C$, then the chromatic number of the intersection graph of $C$ is the same as the chromatic number as the intersection graph of $C \backslash\left\{C_{c}\right\}$. If the curve $C_{c}$ intersects at least one other curve in $C$, then 
let $d$ be a label such that $C_{d}$ intersects $C_{c}$ and $d(c, d)$ is as large as possible. Finally, let $e$ in the cyclic interval $[b, c]$ and $f$ in the cyclic interval $[d, a]$ be such that $C_{e}$ intersects $C_{f}$ and $d(e, f)$ is as large as possible. Properly color the curves that intersect $C_{a}$ with $G(n, k-1)$ colors, the remaining curves that intersect $C_{b}$ with $G(n, k-1)$ colors, the remaining curves that intersect $C_{e}$ with $G(n, k-1)$ colors, and the remaining curves that intersect $C_{f}$ with $G(n, k-1)$ colors. Each of the remaining curves have labels in the cyclic intervals $I_{1}:=[a, b], I_{2}:=[b, e], I_{3}:=[e, f]$, or $I_{4}:=[f, a]$, and no remaining curve with label in $I_{i}$ intersects a remaining curve with label in the interval $I_{j}$ for $1 \leq i<j \leq 4$. Notice that each of the four intervals $I_{1}, I_{2}, I_{3}, I_{4}$ has at most $\left\lfloor\frac{2 n}{3}\right\rfloor$ elements, so $C$ can be properly colored with $G\left(\left\lfloor\frac{2 n}{3}\right\rfloor, k\right)+4 G(n, k-1)$ colors, which completes the proof.

Iterating Lemma 7.4, we have

$$
G(n, k) \leq 4 \sum_{i \geq 0} G\left(\left\lfloor\left(\frac{2}{3}\right)^{i} n\right\rfloor, k-1\right) \leq \frac{4}{\log 2 / 3}(1+\log n) G(n, k-1) .
$$

Trivially, $G(n, 2)=1$. Therefore, there is an absolute constant $c$ such that for $n>1$ and $k>2$, we have

$$
G(n, k) \leq\left(\frac{4}{\log 2 / 3}(1+\log n)\right)^{k-2} G(n, 2) \leq\left(c(\log n)^{k-2}\right),
$$

which, with Lemmas 7.2 and Lemma 7.3, completes the proof of Theorem 7.1 .

\section{Open problems}

A few outstanding unsolved problems related to our subject are listed below.

Problem 8.1 Does the family of intersection graphs of continuous curves in the plane have the Erdös-Hajnal property?

Problem 8.2 Is it true that every perfect graph with $n$ vertices or its complement contains a bi-clique of size $n^{1-o(1)}$ ?

Problem 8.3 Does there exist for every integer $k>2$ a natural number $C_{k}$ with the property that the intersection graph of any finite collection of convex sets in the plane with no $k$ pairwise intersecting members is $C_{k}$-colorable? 
We do not even know if every such intersection graphs with $n$ vertices contains an independent set of size at least $c_{k} n$, for a suitable constant $c_{k}>0$.

A geometric graph is a graph whose vertices are points in the plane in general position and whose edges are straight-line segments connecting certain pairs of points. An affirmative answer to (even the weaker form of) Problem 8.3 would yield that any geometric graph with $n$ vertices and no $k$ pairwise crossing edges has at most $D_{k} n$ edges, where $D_{k}$ is a constant depending only on $k$. This is known to be true for $k \leq 4$; see $[2,47,1]$.

Problem 8.4 [8] Does there exist a positive constant c such that every complete geometric graph with $n$ vertices has cn pairwise crossing edges?

Problem 8.5 [26] Is it true that any $K_{k, k}$-free intersection graph of $n$ segments in $\mathbb{R}^{3}$ has at most $D_{k} n$ edges, for some $D_{k}>0$ depending only on $k$ ?

\section{References}

[1] E. Ackerman, On the maximum number of edges in topological graphs with no four pairwise crossing edges, Proc. 22nd ACM Sympos. on Comput. Geom. ACM Press, 2006, 259-263.

[2] P. K. Agarwal, B. Aronov, J. Pach, R. Pollack, and M. Sharir, Quasiplanar graphs have a linear number of edges, Combinatorica 17 (1997), $1-9$.

[3] P.K. Agarwal and J. Matoušek, Range searching with semialgebraic sets, Discrete Comput. Geom. 11 (1994), 393-418.

[4] M. Ajtai, J. Komlós, and E. Szemerédi, A note on Ramsey numbers, J. Combin. Theory Ser. A 29 (1980), 354-360.

[5] J. Akiyama, K. Hosono, and M. Urabe, Some combinatorial problems, Discrete Math. 116 (1963), 291-298.

[6] N. Alon, Ramsey graphs cannot be defined by real polynomials, J. Graph Theory 14 (6) (1990), 651-661.

[7] N. Alon, J. Pach, R. Pinchasi, R. Radoičić, and M. Sharir, Crossing patterns of semi-algebraic sets, J. Combin. Theory Ser. A 111 (2) (2005), 310-326.

[8] B. Aronov, P. Erdős, W. Goddard, D. Kleitman, M. Klugerman, J. Pach, and L. J.'Schulman, Crossing families, Combinatorica 14 (2) (1994), 127134. 
[9] E. Asplund and B. Grünbaum, On a coloring problem, Math. Scand. 8 (1960), 181-188.

[10] L. Babai, Open problem, in: Proc. 5th Hungar. Conf. Combin. (A. Hajnal and V. T. Sos, eds.), Keszthely, Hungary, 1976, Vol. 2, North Holland (1978), 1189.

[11] B. Barak, A. Rao, R. Shaltiel, and A. Wigderson, 2-source dispersers for sub-polynomial entropy and Ramsey graphs beating the Frankl-Wilson construction, in: Proceedings of STOC 06, (2006), 671-680.

[12] S. Basu, Combinatorial complexity in o-minimal geometry, manuscript, 2006.

http://www.arxiv.org/abs/math.CO/0612050

[13] S. Basu, R. Pollack, and M.-F. Roy, Algorithms in real algebraic geometry. Second edition. Algorithms and Computation in Mathematics 10, Springer-Verlag, Berlin, 2006.

[14] J. P. Burling, On coloring problems of families of prototypes, Ph.D. Thesis, Univ. of Colorado, 1965.

[15] D. Conlon, A new upper bound for diagonal Ramsey numbers, to appear in Annals of Math., 2007.

[16] D. G. Corneil, Y. Perl, and L. K. Stewart, A linear recognition algorithm for cographs, SIAM J. Comput. 14 (1985), 926-934.

[17] R. P. Dilworth, A decomposition theorem for partially ordered sets, Annals of Math. 51 (2) (1950), 161-166.

[18] A. Dumitrescu and G. Tóth, Ramsey-type results for unions of comparability graphs, Graphs Combin. 18 (2002), 245-251.

[19] G. Ehrlich, S. Even, and R. E. Tarjan, Intersection graphs of curves in the plane, J. Combin. Theory Ser. B 21 (1) (1976), 8-20.

[20] P. Erdös, Some remarks on the theory of graphs, Bulletin of the Amer. Math. Soc. 53 (1947), 292-294.

[21] P. Erdős and A. Hajnal, Ramsey-type theorems, Discrete Appl. Math. 25 (1989), 37-52.

[22] P. Erdős, A. Hajnal, and J. Pach, Ramsey-type theorem for bipartite graphs, Geombinatorics 10 (2000), 64-68.

[23] P. Erdős and G. Szekeres, A combinatorial problem in geometry, Compositio Mathematica 2 (1935), 463-470.

[24] J. Fox, A bipartite analogue of Dilworth's theorem, Order 23 (2-3) (2006), 197-209. 
[25] [25] J. Fox and J. Pach, A bipartite analogue of Dilworth's theorem for multiple partial orders, European J. Combinatorics, to appear. http://math.nyu.edu/ pach/publications/multi060406.pdf

[26] J. Fox and J. Pach, Separator theorems and Turán-type results for planar intersection graphs, submitted, 2007.

[27] J. Fox and J. Pach, Coloring planar intersection graphs, manuscript, 2007.

[28] J. Fox, J. Pach, and Cs. D. Tóth, Turán-type results for partial orders and intersection graphs of convex sets, Israel J. Mathematics, to appear.

[29] J. Fox, J. Pach, and Cs. D. Tóth, A bipartite strengthening the Crossing Lemma, Graph Drawing 200\%, LNCS, Springer-Verlag, Berlin, to appear.

[30] J. Fox and B. Sudakov, Density theorems for bipartite graphs and related Ramsey-type results, submitted.

[31] P. Frankl and R. M. Wilson, Intersection theorems with geometric consequences, Combinatorica 1 (4) (1981), 357-368.

[32] I. Karapetian, On coloring of circular arc graphs, Doklady AN ArmSSR (Notes of Armenian Acad. Sci. 70 (1980) (5), 306-311 (in Russian).

[33] I. Karapetian, Chordal Graphs, Matematicheskie voprosi kibernetiki i vichislitelnoi tehniki 14 (1985), 6-10, Erevan (in Russian).

[34] G. Károlyi, J. Pach, and G. Tóth, G. Ramsey-type results for geometric graphs. I, Discrete Comput. Geom. 18 (3) (1997), 247-255.

[35] J. H. Kim, The Ramsey number $R(3, t)$ has order of magnitude $t^{2} / \log t$. Random Structures Algorithms 7 (1995), 173-207.

[36] S. J. Kim, A. Kostochka, and K. Nakprasit, On the chromatic number of intersection graphs of convex sets in the plane, Electron. J. Combin. 11 (2004), \#R52.

[37] P. Koebe, Kontaktprobleme der konformen Abbildung, Berichte über die Verhandlungen der Sachsischen Akademie der Wissenschaften, Leipzig, Mathematische-Physische Klasse 88 (1936), 141-164.

[38] A. Kostochka, Coloring intersection graphs of geometric graphs, in: Towards a Theory of Geometric Graphs (J. Pach Ed.), AMS, Providence, Rhode Island, 127-138, 2003.

[39] A. Kostochka and J. Kratochvíl, Covering and coloring polygon-circle graphs, Discrete Math. 163 (1997), 299-305.

[40] J. Kynčl, unpublished.

[41] D. Larman, J. Matoušek, J. Pach, and J. Töröcsik, A Ramsey-type result for convex sets, Bull. London Math. Soc. 26 (2) (1994), 132-136. 
[42] R. J. Lipton and R. E. Tarjan, A separator theorem for planar graphs, SIAM J. Appl. Math. 36 (2) (1979), 177-189.

[43] S. McGuinness, Colouring arcwise connected sets in the plane. I, Graphs Combin. 16 (2000), 429-439.

[44] S. McGuinness, Colouring arcwise connected sets in the plane. II Graphs Combin. 17 (2001), 135-148.

[45] G. L. Miller, S.-H. Teng, W. Thurston, and S. A. Vavasis, Separators for sphere-packings and nearest neighbor graphs, J. ACM 44 (1) (1997), $1-29$.

[46] J. Pach, Decomposition of multiple packing and covering, 2. Kolloquium über Diskrete Geometrie, Salzburg (1980), 169-178.

[47] J. Pach, R. Radoičić, and G. Tóth, Relaxing planarity for topological graphs, Discrete and Computational Geometry (J. Akiyama, M. Kano, eds.), vol. 2866 of LNCS, Springer-Verlag, Berlin, 2003, 221-232.

[48] J. Pach and J. Solymosi, Crossing patterns of segments, J. Combin. Theory Ser. A 96 (2001), 316-325.

[49] J. Pach and G. Tóth, Comment on Fox News, Geombinatorics 15 (2006), 150-154.

[50] J. Pach and J. Törőcsik, Some geometric applications of Dilworth's theorem, Discrete Comput. Geom. 12 (1) (1994), 1-7.

[51] J. B. Sidney, S. J. Sidney, and J. Urrutia, Circle orders, n-gon orders and the crossing number, Order 5 (1) (1988), 1-10.

[52] H. Tietze, Über das Problem der Nachbargebiete im Raum, Monatshefte Math. 16 (1905), 211-216.

[53] A. C. Yao and F. F. Yao, A general approach to $d$-dimensional geometric queries, Proc. 17th Annu. ACM Sympos. Theory Comput. (1983), 163168. 